\title{
Establishment of the Clone of Gastrointestinal Stromal Tumor Cells with the Signs of Mulliple Drug Resistance and Assessment of tis Properties
}

DOI: 10.17691/stm2016.8.4.05

Received August 2, 2016

R.R. Khusnutdinov, PhD Student, General Pathology Department;

A.R. Galembikova, PhD Student, General Pathology Department;

S. V. Boichuk, MD, DSC, Professor, Head of General Pathology Department

Kazan State Medical University, 49 Butlerova St., Republic of Tatarstan, Kazan, 420012, Russian Federation

The aim of the investigation was to obtain a tumor cell clone of gastrointestinal stromal tumors (GISTs) possessing resistance to chemotherapeutic agents of different mode of action, and to assess its sensitivity to various groups of chemotherapeutic agents.

Materials and Methods. GIST cell line T-1 was used to obtain a clone of chemoresistant GIST cells. The viability assessment of tumor cells was carried out by using i-CELLigence RTCA cell analyzer (ACEA Biosciences, USA). The sensitivity of GIST T-1-29R cell clone to type II topoisomerase inhibitors (doxorubicin and etoposide); chemotherapeutic agents affecting the dynamic state of the spindle microtubules (vinblastine and paclitaxel); hydroxyurea; cisplatin; targeted drug imatinib were evaluated by using the MTT-based assay. Expression of apoptosis, the markers of DNA damage and chemoresistance was examined by immunoblotting.

Results. A clone of GIST T-1 tumor cell line with the signs of chemoresistance (T-1-29R) was obtained after 8-month of cultivation of GIST tumor cells in the presence of the gradually increasing doses of paclitaxel. T-1-29R cells were found to possess resistance to paclitaxel, and cross-resistance to doxorubicin and etoposide, as well. Sensitivity of T-1-29R cells to other chemotherapeutic agents, including imatinib, did not change. Some multiple drug resistance proteins, e.g. MDR-1, were revealed to have an increased expression level in T-1-29R tumor cells when compared to parent T-1 cells.

Conclusion. A clone of GIST T-1-29R cell line possesses phenotypical features of multiple drug resistance, that makes its perspective use for the assessment of GIST chemosensitivity, and for screening of new compounds for their cytotoxic and antitumor activities in vitro and in vivo, as well.

Key words: gastrointestinal stromal tumors; GISTs; tumor cell cloning; paclitaxel; doxorubicin; etoposide; cytotoxicity; resistance.

Despite a long existing point of view about the low efficacy of chemotherapy for patients with gastrointestinal stromal tumors (GISTs) [1,2], recent data indicates GISTS sensitivity to the certain types of chemotherapeutic drugs. For example, it was shown rather good perspectives of some chemotherapeutic drugs to manage the patients with metastatic and inoperable forms of GIST $[3,4]$. The results obtained by our research group also demonstrate that some GIST cell lines are sensitive to the topoisomerase type II inhibitors in vitro and in vivo $[5,6]$. Of note, the chemotherapeutic drugs indicated above were found to be effective against both types of GIST cell lines known to be sensitive and resistant to the targeted therapy by imatinib. Besides, we also found the imatinib to induce sensitization of GIST tumor cells to chemotherapeutic drugs indicated above [7]. One of the potential mechanisms of this phenomenon might be due to ability of imatinib to inhibit the processes of DNA homologous recombination and, as a consequence, attenuate the ability of tumor cells to repair DNA damage, induced by doxorubicin and etoposide [8]. The results of this experimental data correlate with the findings of some clinical investigations, having shown the efficacy of imatinib application in combination with small doses of doxorubicin in patients with GIST [9]. It is noteworthy, that the efficacy of this combination was demonstrated also in the group of patients, in which traditional KIT/PDGFRA mutations were not determined.

The aforementioned testifies to good perspectives of applying some chemotherapeutic drugs to treat patients with GISTs. But the fact of a steady development of malignant neoplasm chemoresistance following the beginning of chemotherapy in cancer patients is quite evident. The time period for the tumor to remain sensitive to chemotherapy depends on many factors and may vary in each individual case. Consequently, an individual assessment of tumor sensitivity to chemotherapeutic drugs and selection of individual appropriate schemes of chemotherapy for cancer patients, including those with GIST, seems to be very promising.

One of the criteria used in searching for novel perspective compounds possessing anti-tumor activity is

For contacts: Sergey V. Boichuk, e-mail: boichuksergei@mail.ru 
related to their ability to induce death of the tumor cells resistant to the known chemotherapeutic drugs. Due to a long existing erroneous, to our opinion, point of view of the low efficacy of chemotherapy to treat GIST patients, there are no currently available GIST cell lines that possess phenotypical features of multiple drug resistance (MDR).

The aim of the study was to obtain a tumor cell clone of gastrointestinal stromal tumors possessing resistance to chemotherapeutic drugs of different mode of action, and to assess its sensitivity to various groups of such chemotherapeutic drugs.

Materials and Methods. GIST T-1 cell line sensitive to the targeted imatinib was chosen as an object of investigation [10]. To obtain a clone of GIST cells with MDR features, tumor cells were cultured with paclitaxel for 8 months. The initial dose of the chemotherapeutic drug in the cell culture was $0.4 \mathrm{nmol}$, the final one amounted to $24 \mathrm{nmol}$. The cells were cultured under standard conditions $\left(37^{\circ} \mathrm{C}, 5 \% \mathrm{CO}_{2}\right)$ in the culture RPMI-1640 medium supplemented with L-glutamine, penicillin and streptomycin (PanEco, Russia), as well as $10 \%$ fetal calf serum (HyClone, USA). Every 2 months a comparative evaluation of sensitivity of GIST cells and their clone to different groups of chemotherapeutic drugs: doxorubicin, vinblastine, paclitaxel, imatinib (Sigma-Aldrich, USA), etoposide, hydroxyurea (Calbiochem, USA), and cisplatin was performed. For this purpose, tumor cells were inoculated into the wells of a flat-bottom 96-well cell culture plate (Corning, USA) and cultured in triplicates for $48-72 \mathrm{~h}$ at $37^{\circ} \mathrm{C}$ in a humidified atmosphere containing $5 \% \mathrm{CO}_{2}$. Cytotoxicity of the above mentioned compounds was estimated colorimetrically by using the CellTiter $96^{\circledR}$ AQueous MTS Reagent Powder (Promega, USA) and Multiscan FC plate analyzer (Thermo Scientific, USA) at $492 \mathrm{~nm}$ wavelength. Proliferative capacity of GIST cells was assessed in the presence of paclitaxel by on-line measurement of the cell index using iCELLigence RTCA cell analyzer (ACEA Biosciences, USA) in accordance with the manufacture's protocol.

The expression level of the proteins, being the markers of DNA damage, apoptosis and chemoresistance, was determined by immunoblotting and appropriate monoclonal antibodies.

The results were statistically analyzed using Student t-test in Microsoft Excel 2007 program.

Results and Discussion. After 8 months of culturing GIST T-1 cells in the presence of gradually

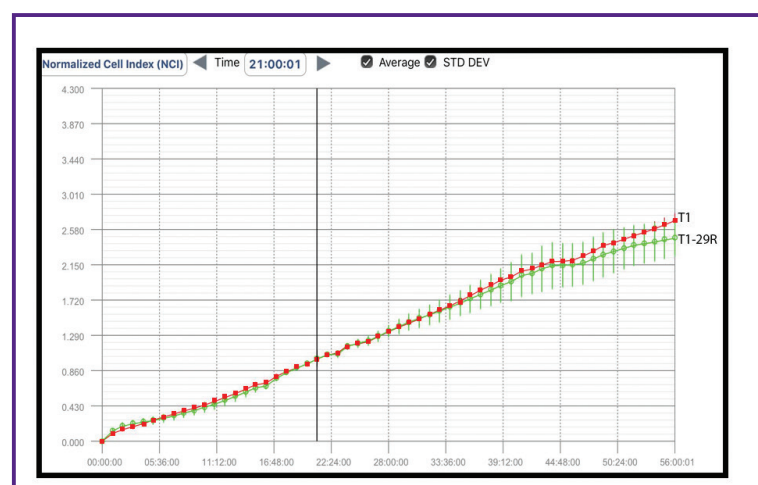

a

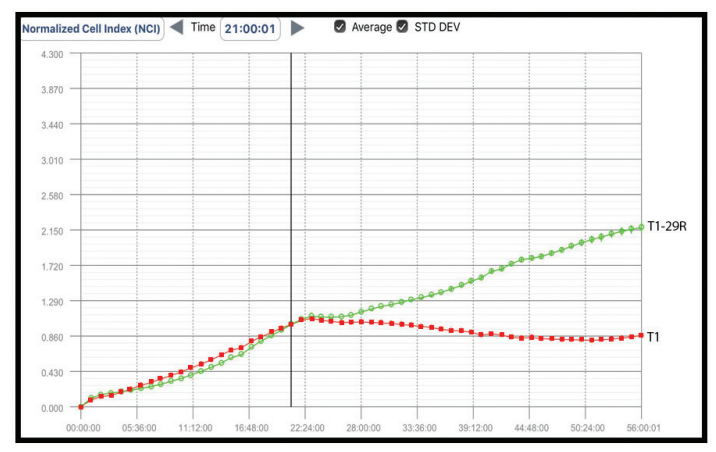

C

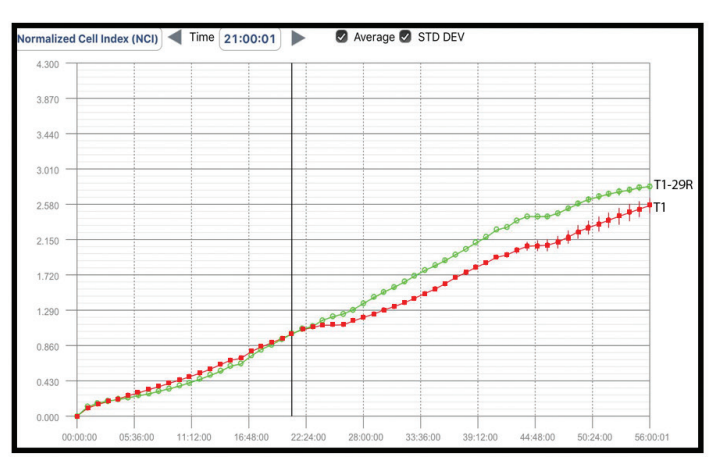

b

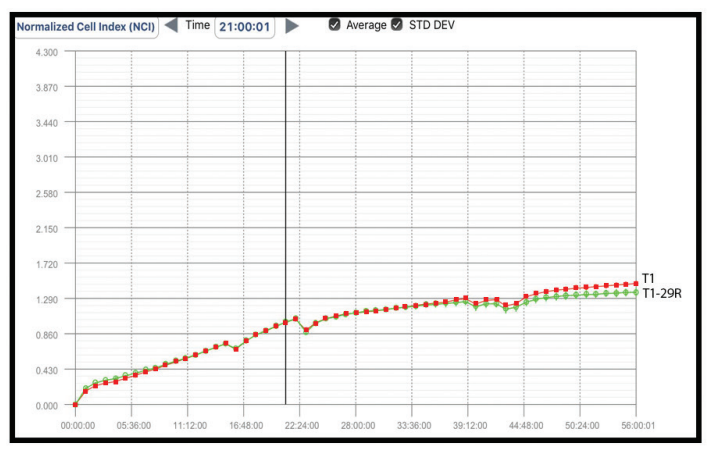

d

Figure 1. Assessment of proliferative capacity of GIST T-1 and T-1-29R cells in the absence of the paclitaxel ((a) control) and its various concentrations: (b) $1 \mathrm{nmol}$; (c) $10 \mathrm{nmol}$; (d) $100 \mathrm{nmol}$. The tumor cell growth curves in vitro were obtained using iCELLigence device 


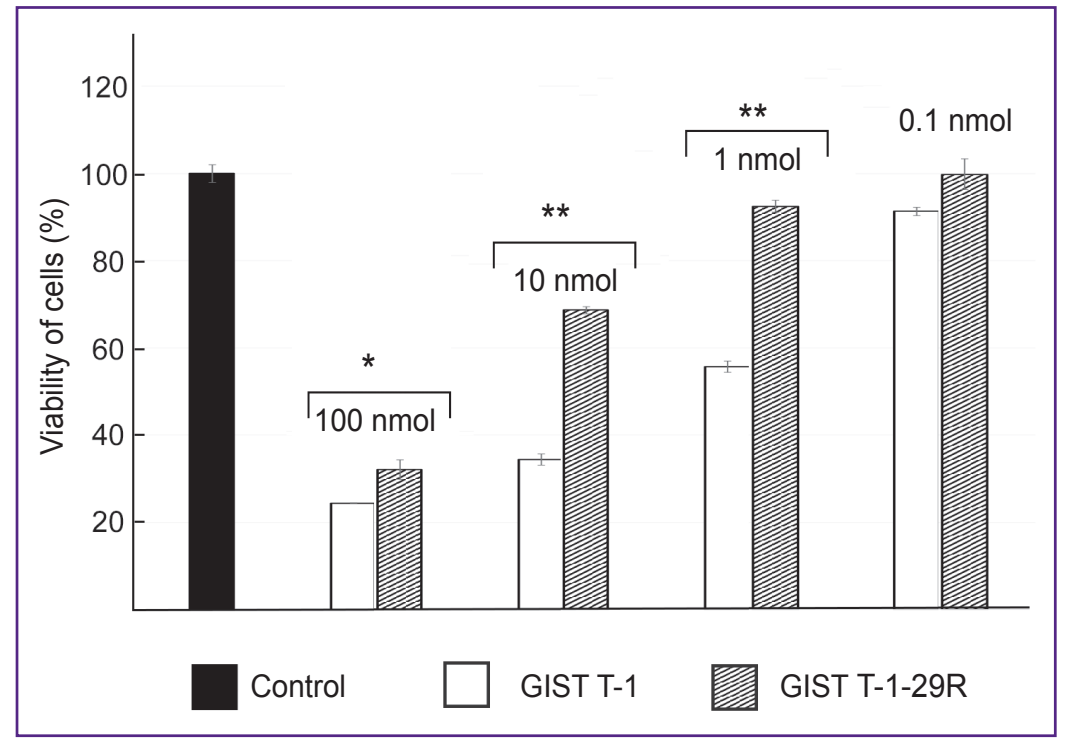

Figure 2. Cytotoxic activity of paclitaxel against GIST T-1 and T-1-29R cells. Cytotoxicity was determined by MTT-based assay. Statistically significant difference of values between the examined cell lines: * $p<0.01$; ${ }^{* *} p<0.001$

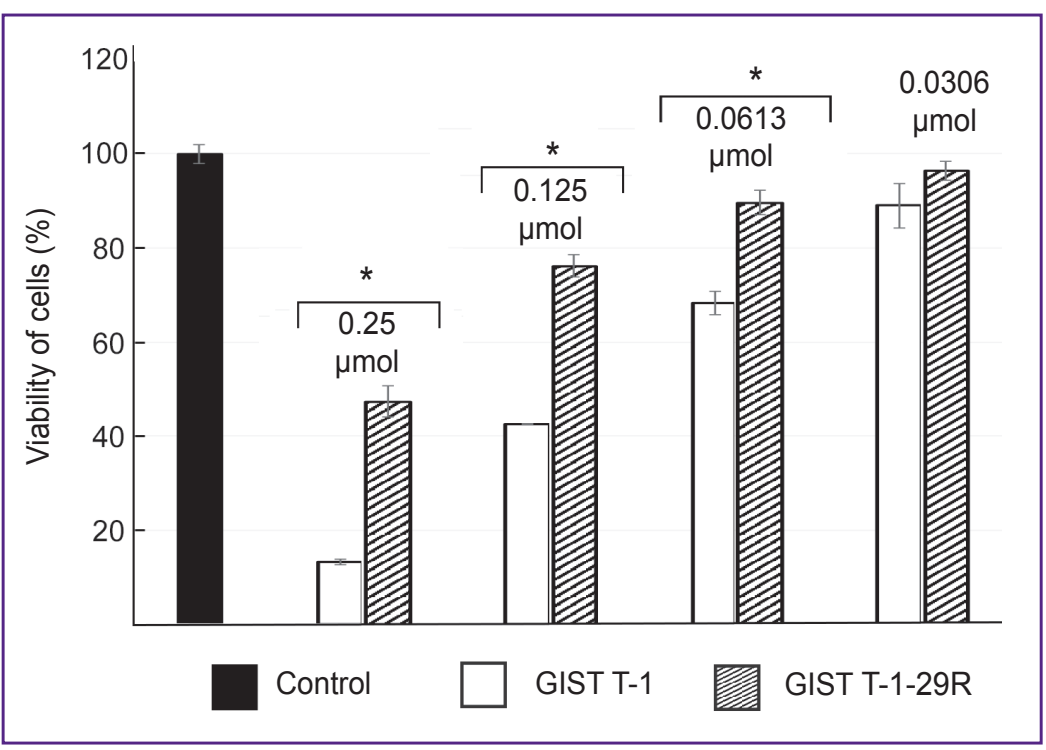

Figure 3. Cytotoxic activity of doxorubicin against GIST T-1 and T-1$29 \mathrm{R}$ cells. Cytotoxicity was determined by MTT-based assay. Statistically significant difference of values between the examined cell lines; * $p<0.001$ growing concentrations of paclitaxel $\left(29^{\text {th }}\right.$ passage), the signs of resistance to the chemotherapeutic drug indicated above were found - the obtained clone of GIST tumor cells was designated as a T-1-29R.

The results of on-line assessment of proliferative activity in the presence of paclitaxel proved the chemoresistance of T-1-29R cells. For example, proliferative capacity of "maternal" and "daughter" cell clones in the absence of this drug (control) was found to be similar (Figure 1, (a)). When T-1-29R were cultured in the presence of paclitaxel in the doses of 1 and $10 \mathrm{nmol}$ (Figure 1 (b), (c), respectively), their proliferation rate was significantly higher when compared to "maternal" GIST T-1 clone. Culturing of "maternal" and "daughter" GIST cells in the presence of high doses of paclitaxel $(100 \mathrm{nmol})$ was fatal to both types of tumor cells (Figure 1 (d)).

Data obtained by using the photocolorimetric MTT-based assay also showed the higher viability of T-129R cells when compared to "maternal" GIST T-1 tumor cells in the presence of the paclitaxel, that indicated the acquired resistance to paclitaxel in GIST T-1-29R cells (Figure 2).

Apart from the resistance to paclitaxel in GIST T-1-29R cells, the resistance was also noted to topoisomerase type II inhibitors, e.g. doxorubicin (Figure 3).

Based on the investigations we determined the effective concentrations of chemotherapeutic agents inducing $50 \%$ inhibition of the survival $\left(\mathrm{IC}_{50}\right)$ of $\mathrm{T}-1$ and T-1-29R tumor cells. Comparative values of $\mathrm{IC}_{50}$ for the chemotherapeutic agents presented in the Table indicate, that GIST clone T-1-29R cells possesses cross-resistance to type II topoisomerase inhibitors, and also to some other chemotherapeutic agents affecting dynamic state of the spindle microtubules (taxanes).

Cytotoxic properties of chemotherapeutic agents against gastrointestinal stromal tumor cells

\begin{tabular}{|lcc|}
\hline \multicolumn{1}{c}{$\begin{array}{c}\text { Chemotherapeutic } \\
\text { agent }\end{array}$} & GIST T-1 & GIST T-1-29R \\
\hline Paclitaxel $(\mathrm{nmol})$ & $0.67 \pm 0.09$ & $21.47 \pm 1.03$ \\
\hline Doxorubicin $(\mu \mathrm{g} / \mathrm{ml})$ & $0.14 \pm 0.07$ & $0.27 \pm 0.03$ \\
\hline Etoposide $(\mu \mathrm{mol})$ & $42.69 \pm 1.38$ & $70.95 \pm 0.97$ \\
\hline Cisplatin $(\mu \mathrm{mol})$ & $5.34 \pm 0.24$ & $6.4 \pm 0.4$ \\
\hline
\end{tabular}

Since apoptosis is believed to be the main mechanism of tumor cells death after the exposure to abovementioned chemotherapeutic agents, an ability of paclitaxel to induce apoptosis of "maternal" (T-1) and "daughter" (T-1-29R) GIST cells was performed. Incubation of "maternal" GIST (T-1) cells with paclitaxel resulted in a significant and dose-dependent increased levels of expression of the cleaved forms of caspase-3 and poly (ADP-ribose) polymerase (PARP), known as a common apoptosis markers (Figure 4 (a)). As we expected, no increase of 
Figure 4. Paclitaxel (PTX) induces apoptosis of GIST T-1 cells (a), but not of T-1-29R cells (b). Cleaved forms of PARP and caspas- 3 (cl. Casp-3) were used as apoptosis markers, an increased expression of $\mathrm{pH} 2 \mathrm{AX}$ and pPLK-1 were used as the markers of M-phase. Paclitaxel concentrations are shown in nmol. Actin expression level was used as a loading control

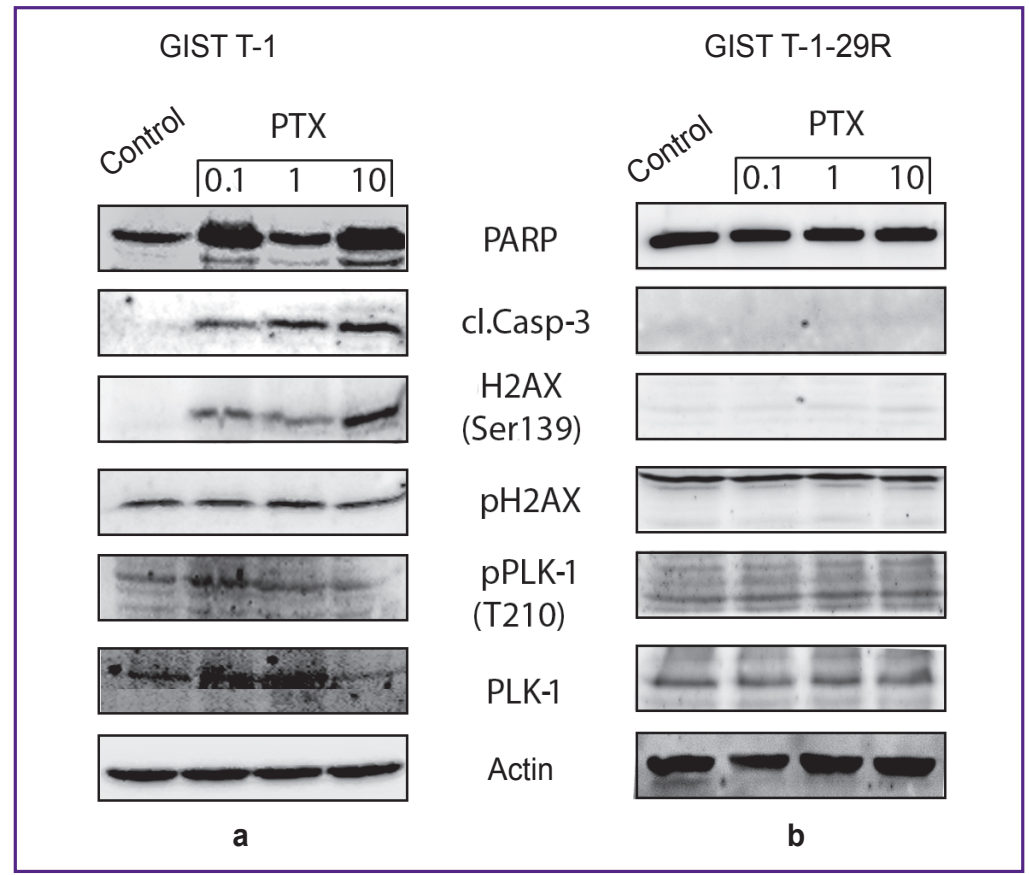

apoptotic markers indicated above was found in T-1-29R cells after paclitaxel exposure (Figure 4 (b)).

Similarly, incubation of GIST T-1 cells (but not T-1-29R) with the topoisomerase type II inhibitors induced a dose-dependent increase of expression levels of the apoptotic markers (Figure $5(a),(b)$, respectively). Of note, the level of histone H2A expression, phosphorylated by serin residue in position 139 (Ser139) (pH2AX), which is a wellknown marker of double-strand DNA breaks, increased significantly in GIST T-1 cells after the exposure to chemotherapeutic agents indicated above (Figure 5 (a)). At the same time, only a minimal increase of $\mathrm{pH} 2 \mathrm{AX}$ expression was detected for T-1-29R cells after the exposure to etoposide (Figure 5 (b)). The effect of doxorubicin was also less efficient when compared to "maternal" GIST T-1 cells.

The decreased expression of apoptotic markers and double-strand breaks in T-1-29R cells after the exposure to topoisomerase type II inhibitors might be due to the numerous factors, such as activation of DNA repair processes after the exposure to genotoxic drugs indicated above, intensification of drug elimination from the tumor cells and, therefore, less time of their action on DNA, etc. Taking into account the time period used to assess the expression level of $\mathrm{pH} 2 \mathrm{AX}$ in our experimental model, the first scenario seems unlikable. A significant increase

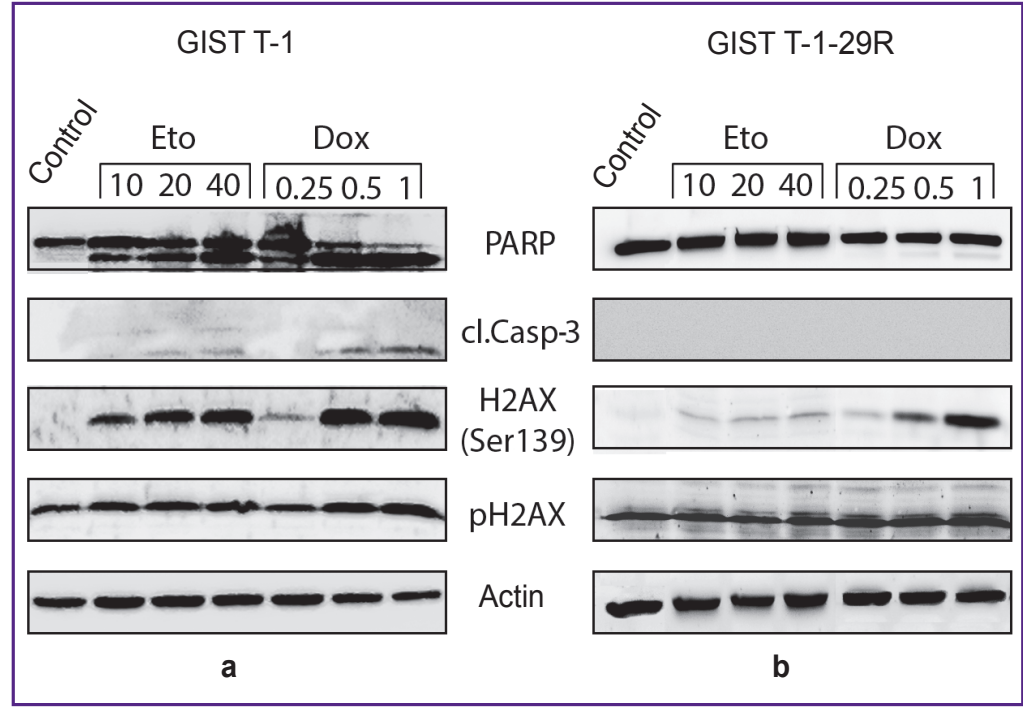

Figure 5. Doxorubicin (Dox) and etoposide (Eto) induce apoptosis in GIST T-1 cells (a), but not in T-1-29R (b). Cleaved forms of PARP and caspas-3 (cl. Casp-3) were used as apoptosis markers, increased expression of pH2AX (Ser139) was used as a marker of DNA double-strand breaks. Concentrations of etoposide are shown in $\mu \mathrm{mol}$, doxorubicin in $\mu \mathrm{g} / \mathrm{ml}$

Figure 6. Dynamics of expression of chemoresistance markers (MDR-1, MRP-1, and ABCG2) in GIST T-1 cells during their culture in the presence of paclitaxel: 1, 2, $3-2,5,8$ months of culture, respectively

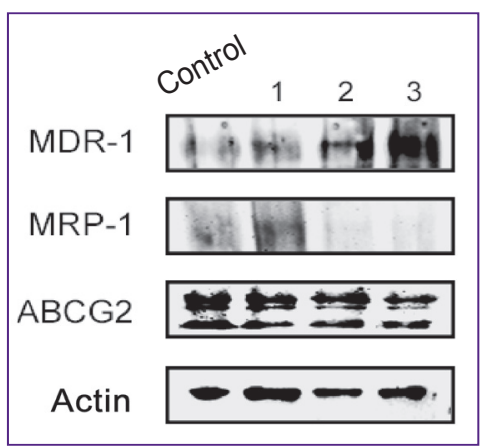


of P-glycoprotein (MDR-1) expression responsible for elimination of cytostatics from the cells and causing, therefore, the development of MDR indicates the high probability for intensification of drug elimination from T-129R tumor cells (Figure 6). Yet, no changes have been noted in the expression levels of some other transporter proteins, responsible for MDR development, e.g. ABCG2 (the basal expression of this proteins was high).

Conclusion. The conducted study enabled us to obtain a clone of GIST T-1 tumor cell line, possessing resistance to the various groups of chemotherapeutic drugs, i.e. influencing the dynamic state of the spindle microtubules (paclitaxel), topoisomerase type II inhibitors (doxorubicin and etoposide). The obtained clone of GIST cells with the features of multiple drug resistance can be further used to screen already existing and newlysynthesized chemical compounds for their cytotoxic and anti-tumor activities against GIST cells. These findings also demonstrate the sensitivity of GIST cells to type II topoisomerase inhibitors and taxanes, which confirms the necessity of revising the existing opinion on GISTs chemoresistance.

Study Funding. The work was supported by the Russian Scientific Fund (grant No.14-15-00342).

Conflicts of Interest. The authors have no conflicts of interest to declare.

\section{References}

1. Verweij J., Casali P.G., Zalcberg J., LeCesne A., Reichardt P., Blay J.Y., Issels R., van Oosterom A., Hogendoorn P.C., Van Glabbeke M., Bertulli R., Judson I. Progression-free survival in gastrointestinal stromal tumours with high-dose imatinib: randomised trial. Lancet 2004; 364(9440): 1127-1134, https://doi.org/10.1016/s01406736(04)17098-0.

2. Dematteo R.P., Heinrich M.C., El-Rifai W.M., Demetri G. Clinical management of gastrointestinal stromal tumors: before and after STI-571. Hum Pathol 2002; 33(5): 466-477, https:// doi.org/10.1053/hupa.2002.124122.

3. Pessetto Z.Y., Weir S.J., Sethi G., Browargd M.A., Godwin A.K. Drug repurposing for gastrointestinal stromal tumor. Mol Cancer Ther 2013; 12(7): 1299-1309, https://doi. org/10.1158/1535-7163.mct-12-0968.

4. Pessetto Z.Y., Ma Y., Hirst J.J., von Mehren M., Weir S.J., Godwin A.K. Drug repurposing identifies a synergistic combination therapy with imatinib mesylate for gastrointestinal stromal tumor. Mol Cancer Ther 2014; 13(10): 2276-2287, https://doi.org/10.1158/1535-7163.MCT-14-0043.

5. Boichuk S., Lee D.J., Mehalek K.R., Makielski K.R., Wozniak A., Seneviratne D.S., Korzeniewski N., Cuevas R., Parry J.A., Brown M.F., Zewe J., Taguchi T., Kuan S.F., Schöffski P., Debiec-Rychter M., Duensing A. Unbiased compound screening identifies unexpected drug sensitivities and novel treatment options for gastrointestinal stromal tumors. Cancer Res 2014; 74(4): 1200-1213, https://doi. org/10.1158/0008-5472.CAN-13-1955.

6. Galembikova A.R., Dunaev P.D., Boichuk S.V. Sensitivity of gastrointestinal stromal tumors (GISTs) to the various chemotherapeutic agents. Sovremennye problemy nauki i obrazovaniya 2015; 6. URL: https://www.scienceeducation.ru/en/article/view?id=23728.

7. Boichuk S.V., Galembikova A.R., Ramazanov B.R., Duensing A. Imatinib enchances the sensitivity of gastrointestinal stromal tumors to topoisomerase II inhibitors. Advances in Molecular Oncology 2015; 2(1): 076, https://doi. org/10.17650/2313-805X.2015.2.1.076-081.

8. Boichuk S.V., Galembikova A.R., Martinova E.V., Ramazanov B.R., Duensing A. Imatinib effectively inhibits homologous recombination and sensitizes gastrointestinal stromal tumor cells to the topoisomerase type II inhibitors. Tsitologiya 2016; 58(3): 178-185.

9. Maurel J., Martins A.S., Poveda A., LópezGuerrero J.A., Cubedo R., Casado A., Martínez-Trufero J., Ramón Ayuso J., Lopez-Pousa A., Garcia-Albeniz X., Garcia del Muro X., de Alava E. Imatinib plus low-dose doxorubicin in patients with advanced gastrointestinal stromal tumors refractory to high-dose imatinib. A phase I-II study by the Spanish Group for Research on Sarcomas. Cancer 2010; 116(15): 3692-3701, https://doi.org/10.1002/cncr.25111.

10. Taguchi T., Sonobe H., Toyonaga S., Yamasaki I., Shuin T., Takano A., Araki K., Akimaru K., Yuri K. Conventional and molecular cytogenetic characterization of a new human cell line, GIST-T1, established from gastrointestinal stromal tumor. Lab Invest 2002; 82(5): 663-665, https://doi. org/10.1038/labinvest.3780461. 